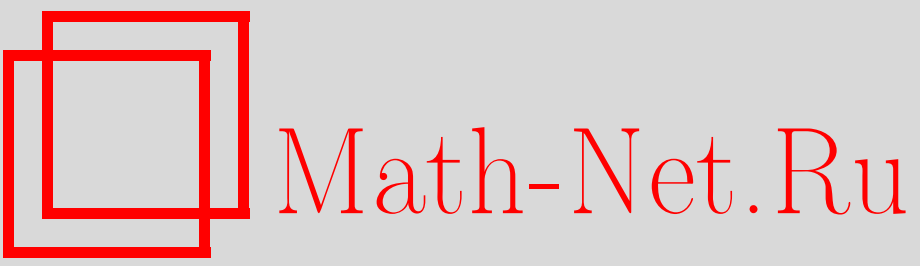

В. И. Манько, С. С. Сафонов, Квантовый осциллятор с трением и классическое представление в квантовой механике, ТМФ, 1997, том 112, номер 3, 467-478

DOI: https://doi.org/10.4213/tmf1056

Использование Общероссийского математического портала Math-Net.Ru подразумевает, что вы прочитали и согласны с пользовательским соглашением

http://www.mathnet.ru/rus/agreement

Параметры загрузки:

IP: 54.162 .127 .20

26 апреля 2023 г., 13:45:24 


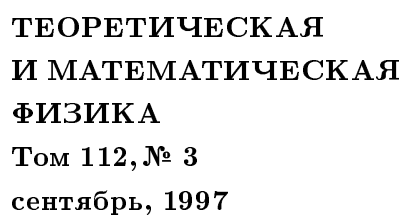

В.И. Манько*, С. С. Сафонов*

\section{КВАНТОВЫЙ ОСЦИЛЛЯТОР С ТРЕНИЕМ И КЛАССИЧЕСКОЕ ПРЕДСТАВЛЕНИЕ В КВАНТОВОЙ МЕХАНИКЕ}

Для квантового осциллятора с трением, который описывается моделью Калдирола-Канаи, вводится маргинальное распределение в рамках классического представления квантовой механики. Данное описание основывается на методе симплектической томографиил. Вычисленное выражение для маргинального распределения осциллятора с трением для когерентного и фоковского состояний выражено через функцию Гаусса и полиномы Эрмита. Кратко описан метод вычисления маргинального распределения с помощью подстановки.

\section{1. ВВЕДЕНИЕ}

Проблема движения с трением занимает особое место в квантовой механике. В рамках квантовой теории сушествует несколько различных моделей, которые описывают трение при движении частицы $[1,2]$. В этих работах матрица плотности гармонического осциллятора с трением была получена с использованием взаимодействия с тепловой баней. В работе [3] было предложено кинетическое уравнение с коэффициентами, которые зависят от трения. Обобшенное уравнение Шредингера с нелинейными членами, описывающими трение, было рассмотрено в [4]. Недавно в [5, 6] обсуждалось другое нелинейное обобщение уравнения Шредингера для описания квантового трения. В нашей работе мы будем использовать простой квантовый гамильтониан, который описывает трение в рамках линейного уравнения Шредингера для волновой функции. Данный гамильтониан был предложен и более подробно рассмотрен в $[7,8]$.

В классической механике движение с трением описывается с помощью уравнения движения. Поэтому в классической механике не существует той неоднозначности, которая присутствует в квантовом описании трения. Недавно в работах $[9,10]$ было предложено классическое представление квантовой механики. Данное представление использует “томографию” как метод для измерения квантового состояния [11-13]. Таким образом, описание квантового движения с делано похожим на описание классического движения, при котором взаимодействие частицы с термостатом описывается положительной функцией распределения. Принимая во внимание возможность классического описания квантовой системы, которое подробно рассмотрено в [9], можно ожидать, что проблему квантового трения можно будет легче понять с точки зрения этого описания. Поэтому цель

\footnotetext{
* Физический институт им. П.Н. Лебедева РАН, Москва, Россия
} 
нашей работы - дать обзор нового классического представления стандартной квантовой механики, предложенного в [9], и обсудить проблему трения в квантовой механике, используя это классическое представление.

Предыдушие попытки дать классическое представление квантовых состояний привели к введению таких квазираспределений в фазовом пространстве системы, как функция Вигнера [14] и квазивероятность Глаубера-Сударшана $[15,16]$. Хотя эти квазивероятности, задающие квантовое состояние, и похожи на положительные функции распределения для некоторых квантовых состояний, они принимают отрицательные значения для многих квантовых состояний и тем самым не описывают вероятность в обычном ее определении. Функция квазивероятности Хусими-Кано $[17,18]$, зависящая от двух переменных $q$ и $p$, нормирована и принимает только положительные значения. В этой связи она могла бы интерпретироваться как распределение вероятности, однако эта функция не есть функция распределения в фазовом пространстве системы, поскольку ее аргументы $q$ и $p$ не являются одновременно измеримыми величинами (координатой и импульсом) в силу соотношения неопределенности.

Использование маргинального распределения для описания квантового состояния основано на соотношении матрицы плотности с характеристической функцией $[12,13]$. Маргинальное распределение для координаты в ансамбле канонически преобразованных систем отсчета в классическом фазовом пространстве было изученно в работах [9-11]. Рассмотренное в этих статьях классическое представление является модифицированным методом оптической томографиии, предложенным в [12]. В оптической томографии, чтобы получить из маргинального распределения функцию Вигнера, было использовано преобразование Радона [12]. Поэтому в нашем случае квантового трения важно знать маргинальное распределение, поскольку можно вычислить функцию Вигнера с помощью измерения этого маргинального распределения. Функция Вигнера и маргинальное распределение в схеме подхода [9-11] связаны при помоши преобразования Фурье, что было подробно продемонстрировано в [11]. По сушеству, томографические схемы для измерения квантового состояния используют новое представление в квантовой механике, отличающееся от представлений Вигнера [14], Глаубера-Сударшана $[15,16]$ и Хусими-Кано $[17,18]$ тем, что состоянию системы в этом представлении взаимно одназначно сопоставляется не квазираспределение, а настоящая функция распределения вероятностей измеримой физической величины.

Для описания квантового осциллятора с трением мы применяем гамильтониан Калдирола-Канаи, который содержит коэффициенты, зависящие от времени $[7,8]$. Обзор решений различных квантовых задач с нестационарными квадратичными гамильтонианами, коэффициенты которых зависят от времени, дан в [19]. Свойства локализованных волновых пакетов для уравнения Дирака, при построении которых используется анализ нестационарных гамильтонианов, изучены в [20]. Поскольку данный гамильтониан содержит зависящие от времени экспоненциальные множители, волновые функции квантового осциллятора с трением также содержат экспоненциальные коэффициенты и множители, зависяшие от времени, что несколько усложняет работу с ними по сравнению с волновыми функциями гармонического осциллятора с трением $[7,8,21]$. Цель настоящей работы - получить маргинальное распределение для квантового осциллятора с постоянной частотой и в отсутствие вынуждаюшей силы при наличии трения и сравнить найденное выражение с недавно полученным маргинальным распределением для обычного гармонического осциллятора $[9,11]$. При вычислении маргинального рас- 
пределения мы выведем и будем использовать производящую функцию для дискретного маргинального распределения, чтобы избежать вычисления функции Вигнера осциллятора с трением в соответствуюших фоковских состояниях. Кроме того, в нашей статье приведен результат вычисления вероятности вызванных трением переходов гармонического осциллятора в рамках классического представления квантовой механики. Найденньй результат сравнивается с результатами других статей $[22,23]$, где обсуждались переходы между дискретными состояниями осциллятора, обусловленные нестационарностью гамильтониана. Мы рассматриваем разные способы нахождения маргинального распределения для осциллятора с трением, а также представляем способ вычисления маргинального распределения из альтернативного уравнения Шредингера $[9,11]$ и способ вычисления с помошью введения подстановки в известное начальное маргинальное распределение [10].

\section{2. ВЫЧИСЛЕНИЕ МАРГИНАЛЬНОГО РАСПРЕДЕЛЕНИЯ ДЛЯ СОСТОЯНИЯ С ПОТЕРЯННОЙ ЭНЕРГИЕЙ}

Как было показано в $[9,11]$, для линейной комбинации координат в фазовом пространстве осциллятора, которая является наблюдаемой величиной

$$
\widehat{X}=\mu \hat{q}+\nu \hat{p}
$$

где $\hat{q}$ - координата, $\hat{p}$ - импульс, а $\mu$ и $\nu$ - действительные числа, распределение $w(X, \mu, \nu)$ связано с состоянием квантовой системы следующим образом:

$$
w(X, \mu, \nu, t)=\int \exp [-i k(X-\mu q-\nu p)] W(q, p, t) \frac{d k d q d p}{(2 \pi)^{2}} .
$$

Распределение $w(X, \mu, \nu, t)$ зависит от двух действительных параметров $\mu, \nu$, а состояние квантовой системы задано функцией Вигнера $W(q, p, t)$. Функция Вигнера нашей системы связана с матрицей плотности в координатном представлении (см., например, [24]) с помошью формулы

$$
W(q, p, t)=\int \rho\left(q+\frac{u}{2}, q-\frac{u}{2}, t\right) \exp [-i p u] d u,
$$

где $\rho\left(q, q^{\prime}, t\right)$ - матрица плотности. Рассмотрим случай квантового осциллятора с трением более конкретно. В работе [21] было показано, что для системы с гамильтонианом Калдирола-Канаи (записанным при $\hbar=m=1$ )

$$
\widehat{H}(t)=\frac{\hat{p}^{2}}{2} \exp (-2 \gamma t)+\omega^{2} \exp (2 \gamma t) \frac{\hat{q}^{2}}{2},
$$

где $\gamma$ (коэффищиент трения) и $\omega$ (собственная частота квантового осциллятора) - постоянные величины, волновые функции для когерентного $|\alpha\rangle$ и фоковского $|n\rangle$ состояний с потерянной энергией могут быть написаны в предположении, что $\omega=1$ :

$$
\begin{gathered}
\Psi_{\alpha}(q, t)=\frac{1}{\sqrt[4]{\pi} \sqrt{\varepsilon}} \exp \left(\frac{i \dot{\varepsilon} e^{2 \gamma t}}{2 \varepsilon} q^{2}+\frac{\sqrt{2} \alpha}{\varepsilon} q-\frac{\dot{\varepsilon}^{*}}{2 \varepsilon} \alpha^{2}-\frac{|\alpha|^{2}}{2}\right), \\
\Psi_{n}(q, t)=\frac{1}{\sqrt[4]{\pi} \sqrt{\varepsilon}}\left(\frac{\varepsilon^{*}}{2 \varepsilon}\right)^{\frac{n}{2}} \frac{1}{\sqrt{n !}} \exp \left(\frac{i \dot{\varepsilon} e^{2 \gamma t}}{2 \varepsilon} q^{2}\right) H_{n}\left(\frac{q}{\sqrt{\varepsilon \varepsilon^{*}}}\right), \quad n=0,1,2, \ldots
\end{gathered}
$$


В этих формулах функция $\varepsilon(t)$ удовлетворяет уравнению

$$
\ddot{\varepsilon}(t)+2 \gamma \dot{\varepsilon}(t)+\varepsilon(t)=0
$$

и начальным условиям

$$
\varepsilon(0)=\frac{1}{\sqrt{\Omega}}, \quad \dot{\varepsilon}(0)=\frac{i \Omega-\gamma}{\sqrt{\Omega}} .
$$

С классическим уравнением движения (7) связан вронскиан, значение которого определяется начальными условиями (8):

$$
\varepsilon^{*} \dot{\varepsilon}-\varepsilon \dot{\varepsilon}^{*}=2 i \exp (-2 \gamma t)
$$

Решение $\varepsilon(t)$ уравнения (7) имеет вид

$$
\varepsilon(t)=\frac{1}{\sqrt{\Omega}} e^{-\gamma t}[\cos (\Omega t)+i \sin (\Omega t)]
$$

где

$$
\Omega^{2}=1-\gamma^{2}
$$

- обобщенная частота квантового осциллятора. Запишем интеграл (2), определяющий маргинальное распределение (используя формулы (3) и определение матрицы плотности), в виде

$$
\begin{aligned}
w(X, \mu, \nu, t)= & \int \exp [-i k(X-\mu q-\nu p)] \Psi\left(q+\frac{u}{2}, t\right) \Psi^{*}\left(q-\frac{u}{2}, t\right) \times \\
& \times \exp (-i p u) \frac{d k d u d q d p}{(2 \pi)^{2}}
\end{aligned}
$$

где $\Psi(q, t)$ - волновая функция (5) или $(6)$, а $\Psi^{*}(q, t)$ - комплексно-сопряженное значение волновой функции $\Psi(q, t)$. Используя преобразование Фурье и основное свойство $\delta$-функции, мы можем написать окончательную форму маргинального распределения для произвольной волновой функции:

$$
w(X, \mu, \nu, t)=\int \exp [-i k X+i k \mu q] \Psi\left(q+\frac{k \nu}{2}, t\right) \Psi^{*}\left(q-\frac{k \nu}{2}, t\right) \frac{d k d q}{(2 \pi)} .
$$

Как известно (см., например, [24]), волновая функция когерентного состояния разлагается в ряд по волновым функциям фоковских состояний:

$$
\Psi_{\alpha}(q, t)=\exp \left(-\frac{|\alpha|^{2}}{2}\right) \sum_{n=0}^{\infty} \frac{\alpha^{n} \Psi_{n}(q, t)}{\sqrt{n !}}
$$

где $\alpha$ - комплексное число. Следовательно, можно получить маргинальное распределение для когерентного состояния как ряд по переменным $\alpha$ и $\alpha^{*}$ :

$$
w_{\alpha}=\exp \left(-\frac{|\alpha|^{2}}{2}\right) \sum_{n, m=0}^{\infty} \frac{\alpha^{n} \alpha^{* m} w_{n m}}{\sqrt{n !} \sqrt{m !}}
$$


где

$$
\begin{aligned}
w_{n m}(X, \mu, \nu, t)= & \int \exp [-i k(X-\mu q-\nu p)] \Psi_{n}\left(q+\frac{u}{2}, t\right) \Psi_{m}^{*}\left(q-\frac{u}{2}, t\right) \times \\
& \times \exp (-i p u) \frac{d k d u d q d p}{(2 \pi)^{2}} .
\end{aligned}
$$

В случае, если $m=n$, функция $w_{n m}$ совпадает с маргинальным распределением фоковского состояния (т.е. $w_{n n} \equiv w_{n}$ ). Подставляя выражение для волновой функции когерентного состояния (5) в интеграл (13) и вычисляя его (используя известный интеграл от гауссиана), мы находим маргинальное распределение для когерентного состояния осциллятора с трением:

$$
\begin{aligned}
w_{\alpha}= & \frac{1}{\sqrt{\pi \varepsilon \varepsilon^{*}\left(a^{2}+b^{2}\right)}} \exp \left(-|\alpha|^{2}\right) \exp \left(-\frac{X^{2}}{\varepsilon \varepsilon^{*}\left(a^{2}+b^{2}\right)}\right) \times \\
& \times \exp \left[-\alpha^{2} \frac{\varepsilon^{* 2}(a-i b)^{2}}{2 \varepsilon \varepsilon^{*}\left(a^{2}+b^{2}\right)}+\alpha \frac{\sqrt{2} \varepsilon^{*} X(a-i b)}{\varepsilon \varepsilon^{*}\left(a^{2}+b^{2}\right)}\right] \times \\
& \times \exp \left[-\alpha^{* 2} \frac{\varepsilon^{2}(a+i b)^{2}}{2 \varepsilon \varepsilon^{*}\left(a^{2}+b^{2}\right)}+\alpha^{*} \frac{\sqrt{2} \varepsilon X(a+i b)}{\varepsilon \varepsilon^{*}\left(a^{2}+b^{2}\right)}\right] .
\end{aligned}
$$

В формуле (17) были введены обозначения

$$
\begin{gathered}
a=\frac{\exp (2 \gamma t) \nu\left(\varepsilon^{*} \dot{\varepsilon}+\varepsilon \dot{\varepsilon}^{*}\right)}{2 \varepsilon \varepsilon^{*}}+\mu, \\
b=\frac{\nu}{\varepsilon \varepsilon^{*}}
\end{gathered}
$$

а функция $\varepsilon(t)$ определена формулой (10). Полученный результат содержит две экспоненты, в аргументах которых находятся $\alpha^{2}, \alpha$ и $\left(\alpha^{*}\right)^{2}, \alpha^{*}$, экспоненту с аргументом $|\alpha|^{2}$, экспоненту, которая не зависит от переменных $\alpha, \alpha^{*}$, и коэффициент, который также не зависит от переменных $\alpha, \alpha^{*}$. Функция $w_{\alpha} e^{|\alpha|^{2}}$ может быть разложена в двойной ряд по переменным $\alpha$ и $\alpha^{*}$. Рассматривая экспоненты с переменными $\alpha^{2}\left(\left(\alpha^{*}\right)^{2}\right)$ и $\alpha\left(\alpha^{*}\right)$ как производящие функции для полиномов Эрмита $H_{n}$ и применяя формулу (15), мы находим маргинальное распределение для фоковского состояния $|n\rangle$

$$
w_{n}(X, \mu, \nu, t)=w_{0}(X, \mu, \nu, t) \frac{1}{2^{n} n !} H_{n}^{2}\left(\frac{X}{\sqrt{\varepsilon \varepsilon^{*}\left(a^{2}+b^{2}\right)}}\right)
$$

где $w_{0}(X, \mu, \nu, t)$ - основное состояние осциллятора

$$
w_{0}(X, \mu, \nu, t)=\frac{1}{\sqrt{\pi \varepsilon \varepsilon^{*}\left(a^{2}+b^{2}\right)}} \exp \left(-\frac{X^{2}}{\varepsilon \varepsilon^{*}\left(a^{2}+b^{2}\right)}\right) .
$$

Сравним найденное выражение для маргинального распределения квантового осциллятора с трением с маргинальным распределением гармонического осциллятора, которое 
было получено в [11]. Чтобы сделать это, рассмотрим маргинальное распределение (20) и $(21)$ в случае $\gamma=0$. Поскольку

$$
\begin{aligned}
& a_{\gamma=0}=\mu, \\
& b_{\gamma=0}=\nu,
\end{aligned}
$$

маргинальное распределение для состояния $|n\rangle$ при $\gamma=0$ выглядит следующим образом:

$$
w_{n}(X, \mu, \nu, t)_{\gamma=0}=w_{0}(X, \mu, \nu, t)_{\gamma=0} \frac{1}{2^{n} n !} H_{n}^{2}\left(\frac{X}{\sqrt{\mu^{2}+\nu^{2}}}\right),
$$

где маргинальное распределение для основного состояния имеет вид

$$
w_{0}(X, \mu, \nu, t)_{\gamma=0}=\frac{1}{\sqrt{\pi\left(\mu^{2}+\nu^{2}\right)}} \exp \left(-\frac{X^{2}}{\left(\mu^{2}+\nu^{2}\right)}\right) .
$$

Сравнивая полученное выражение с результатом работы [11], можно заключить, что маргинальное распределение квантового осциллятора с трением для фоковского состояния с потерянной энергией в пределе $\gamma=0$ совпадает с маргинальным распределением стационарного состояния гармонического осциллятора без трения.

\section{3. КЛАССИЧЕСКОЕ УРАВНЕНИЕ И ИНТЕГРАЛЫ ДВИЖЕНИЯ ДЛЯ МАРГИНАЛЬНОГО РАСПРЕДЕЛЕНИЯ}

В работе [9] было введено эволюшионное уравнение для маргинального распределения как альтернативное уравнение Шредингера для волновой функции (матрицы плотности). Для системы с гамильтонианом, в котором потенциал зависит от времени:

$$
\widehat{H}(t)=\frac{\hat{p}^{2}}{2}+\widehat{V}(q, t),
$$

уравнение эволюции для маргинального распределения имеет вид

$$
\dot{w}-\mu \frac{\partial}{\partial \nu} w-i\left[V\left(-\frac{1}{\partial / \partial X} \frac{\partial}{\partial \mu}-i \frac{\nu}{2} \frac{\partial}{\partial X}, t\right)-V\left(-\frac{1}{\partial / \partial X} \frac{\partial}{\partial \mu}+i \frac{\nu}{2} \frac{\partial}{\partial X}, t\right)\right] w=0 .
$$

Можно показать, что у осциллятора с трением это уравнение принимает форму

$$
\dot{w}-\mu \frac{\partial}{\partial \nu} w-i\left[\widetilde{V}\left(-\frac{1}{\partial / \partial X} \frac{\partial}{\partial \mu}-i \frac{\nu}{2} \frac{\partial}{\partial X}, t^{\prime}\right)-\widetilde{V}\left(-\frac{1}{\partial / \partial X} \frac{\partial}{\partial \mu}+i \frac{\nu}{2} \frac{\partial}{\partial X}, t^{\prime}\right)\right] w=0,
$$

где

$$
\begin{gathered}
\widetilde{V}\left(q, t^{\prime}\right)=\exp \left(2 \gamma t\left(t^{\prime}\right)\right) V\left(q, t\left(t^{\prime}\right)\right)=\exp \left(4 \gamma t\left(t^{\prime}\right)\right) \frac{q^{2}}{2}, \\
t\left(t^{\prime}\right)=-\frac{\ln \left(1-2 \gamma t^{\prime}\right)}{2 \gamma} .
\end{gathered}
$$


Точка в уравнении (28) обозначает производную по переменной $t^{\prime}$. Учитывая новое значение потенциала для осциллятора с трением (29), можно переписать равенство (28) следующим образом:

$$
\frac{\partial}{\partial t^{\prime}} w-\mu \frac{\partial}{\partial \nu} w+\exp (4 \gamma t) \nu \frac{\partial}{\partial \mu} w=0 .
$$

Можно проверить, что найденные выше маргинальные распределения $w_{\alpha}(17)$ и $w_{n}(20)$ удовлетворяют данному уравнению для произвольных $\alpha$ и $n$.

Обсудим теперь проблему интегралов движения для осциллятора с трением. Квантовый осциллятор с трением имеет следуюшие линейные интегралы движения [21]:

$$
\hat{a}(t)=\frac{i}{\sqrt{2}}[\varepsilon(t) \widehat{p}-\dot{\varepsilon}(t) \exp (2 \gamma t) \hat{q}]
$$

и

$$
\hat{a}^{\dagger}(t)=-\frac{i}{\sqrt{2}}\left[\varepsilon^{*}(t) \hat{p}-\dot{\varepsilon}^{*}(t) \exp (2 \gamma t) \hat{q}\right] .
$$

Эти линейные интегралы движения можно переписать, используя переменные $X, \mu, \nu$, в виде

$$
\begin{aligned}
a(t) & =\frac{1}{\sqrt{2}}\left[\frac{\partial}{\partial X}\left(\frac{\varepsilon \mu}{2}+\frac{\dot{\varepsilon} e^{2 \gamma t} \nu}{2}\right)+i\left(\frac{\partial}{\partial X}\right)^{-1}\left(\dot{\varepsilon} e^{2 \gamma t} \frac{\partial}{\partial \mu}-\varepsilon \frac{\partial}{\partial \nu}\right)\right], \\
a^{\dagger}(t) & =-\frac{1}{\sqrt{2}}\left[\frac{\partial}{\partial X}\left(\frac{\varepsilon^{*} \mu}{2}-\frac{\dot{\varepsilon}^{*} e^{2 \gamma t} \nu}{2}\right)+i\left(\frac{\partial}{\partial X}\right)^{-1}\left(\dot{\varepsilon}^{*} e^{2 \gamma t} \frac{\partial}{\partial \mu}+\varepsilon^{*} \frac{\partial}{\partial \nu}\right)\right] .
\end{aligned}
$$

Для того чтобы получить эти формы операторов, мы использовали связь, возникаюшую при действии линейных интегралов движения на матрицу плотности $\rho\left(X, X^{\prime}, t\right)$, функцию Вигнера $W(q, p, t)$ и маргинальное распределение $w(X, \mu, \nu, t)$ (см., например, [25]).

Рассмотрим операторы

$$
\hat{a}^{\dagger}(t) \hat{a}(t)=\frac{1}{2}\left[\varepsilon \varepsilon^{*} \hat{p}^{2}-\varepsilon^{*} \dot{\varepsilon} \exp (2 \gamma t) \hat{p} \hat{q}-\dot{\varepsilon}^{*} \varepsilon \exp (2 \gamma t) \hat{q} \hat{p}+\dot{\varepsilon}^{*} \dot{\varepsilon} \exp (4 \gamma t) \hat{q}^{2}\right]
$$

и

$$
\left(\hat{a}^{\dagger}(t) \hat{a}(t)\right)^{*}=\frac{1}{2}\left[\varepsilon \varepsilon^{*} \hat{p}^{2}-\varepsilon^{*} \dot{\varepsilon} \exp (2 \gamma t) \hat{q} \hat{p}-\dot{\varepsilon}^{*} \varepsilon \exp (2 \gamma t) \hat{p} \hat{q}+\dot{\varepsilon}^{*} \dot{\varepsilon} \exp (4 \gamma t) \hat{q}^{2}\right]
$$

Здесь знак * обозначает комплексно-сопряженный оператор. Эти операторы также являются интегралами движения. Они были обобшены для случая осциллятора с трением с помошью интегралов движения Ермакова, которые были найдены для классического осциллятора с переменной частотой в работе [26] и заново получены для квантового параметрического осциллятора в [27]. Поступая так же, как с операторами (32) и (33), 
переписываем операторы (36) и (37) в переменных маргинального распределения в виде

$$
\begin{aligned}
a^{\dagger}(t) a(t)= & \frac{1}{2}\left\{\left(\frac{\partial}{\partial X}\right)^{-2}\left[\varepsilon \varepsilon^{*}\left(\frac{\partial}{\partial \nu}\right)^{2}+\dot{\varepsilon}^{*} e^{4 \gamma t}\left(\frac{\partial}{\partial \mu}\right)^{2}-e^{2 \gamma t}\left(\varepsilon^{*} \dot{\varepsilon}+\varepsilon \dot{\varepsilon}^{*}\right) \frac{\partial^{2}}{\partial \mu \partial \nu}\right]-\right. \\
& -\left(\frac{\partial}{\partial X}\right)^{2}\left[\varepsilon \varepsilon^{*} \mu^{2}+\dot{\varepsilon} \dot{\varepsilon}^{*} e^{4 \gamma t} \nu^{2}+e^{2 \gamma t}\left(\varepsilon^{*} \dot{\varepsilon}+\varepsilon \dot{\varepsilon}^{*}\right) \mu \nu\right]- \\
& -i e^{2 \gamma t}\left[\frac{\mu}{2}\left(\varepsilon^{*} \dot{\varepsilon}+\varepsilon \dot{\varepsilon}^{*}\right)+e^{2 \gamma t} \dot{\varepsilon}^{*} \dot{\varepsilon} \nu\right] \frac{\partial}{\partial \mu}+ \\
& \left.+i\left[\frac{\left(\varepsilon^{*} \dot{\varepsilon}+\varepsilon \dot{\varepsilon}^{*}\right)}{2} e^{2 \gamma t} \nu+\varepsilon \varepsilon^{*} \mu\right] \frac{\partial}{\partial \nu}-1\right\}
\end{aligned}
$$

и

$$
\begin{aligned}
\left(a^{\dagger}(t) a(t)\right)^{*}= & \frac{1}{2}\left\{\left(\frac{\partial}{\partial X}\right)^{-2}\left[\varepsilon \varepsilon^{*}\left(\frac{\partial}{\partial \nu}\right)^{2}+\dot{\varepsilon}^{*} e^{4 \gamma t}\left(\frac{\partial}{\partial \mu}\right)^{2}+e^{2 \gamma t}\left(\varepsilon^{*} \dot{\varepsilon}+\varepsilon \dot{\varepsilon}^{*}\right) \frac{\partial^{2}}{\partial \mu \partial \nu}\right]-\right. \\
& -\left(\frac{\partial}{\partial X}\right)^{2}\left[\varepsilon \varepsilon^{*} \mu^{2}+\dot{\varepsilon} \dot{\varepsilon}^{*} e^{4 \gamma t} \nu^{2}-e^{2 \gamma t}\left(\varepsilon^{*} \dot{\varepsilon}+\varepsilon \dot{\varepsilon}^{*}\right) \mu \nu\right]+ \\
& +i e^{2 \gamma t}\left[\frac{\mu}{2}\left(\varepsilon^{*} \dot{\varepsilon}+\varepsilon \dot{\varepsilon}^{*}\right)-e^{2 \gamma t} \dot{\varepsilon}^{*} \dot{\varepsilon} \nu\right] \frac{\partial}{\partial \mu}+ \\
& \left.+i\left[\frac{\left(\varepsilon^{*} \dot{\varepsilon}+\varepsilon \dot{\varepsilon}^{*}\right)}{2} e^{2 \gamma t} \nu-\varepsilon \varepsilon^{*} \mu\right] \frac{\partial}{\partial \nu}+1\right\}
\end{aligned}
$$

Для того чтобы получить данные операторы, мы использовали уже упомянутую выше связь, возникающую при действии операторов $a$ и $a^{\dagger}$ на матрицу плотности $\rho\left(X, X^{\prime}, t\right)$, функцию Вигнера $W(q, p, t)$ и маргинальное распределение $w(X, \mu, \nu, t)$. Рассматривая, как операторы (32) и (33) действуют на матрицу плотности, и учитывая связь матрищы плотности с маргинальным распределением (т.е. используя операторы (34) и (35)), мы получаем для маргинального распределения $w_{\alpha}$ когерентного состояния $|\alpha\rangle(17)$ следующие уравнения:

$$
a(t) w_{\alpha}(X, \mu, \nu, t)=\alpha w_{\alpha}(X, \mu, \nu, t)
$$

и

$$
a^{\dagger}(t) w_{\alpha}(X, \mu, \nu, t)=\alpha^{*} w_{\alpha}(X, \mu, \nu, t) .
$$

Аналогично, рассматривая операторы $a^{\dagger} a$ и $\left(a^{\dagger} a\right)^{*}$ (уравнения (38) и (39)), получаем для маргинального распределения $w_{n}$ фоковского состояния $|n\rangle$ (20) следующие уравнения:

$$
\begin{aligned}
a^{\dagger}(t) a(t) w_{n}(X, \mu, \nu, t) & =n w_{n}(X, \mu, \nu, t), \\
\left(a^{\dagger}(t) a(t)\right)^{*} w_{n}(X, \mu, \nu, t) & =n w_{n}(X, \mu, \nu, t),
\end{aligned}
$$

и, таким образом, маргинальное распределение (17) является решением системы уравнений (40), (41), а распределение (20) - решением системы уравнений (42), (43). 
КВАНТОВЫЙ ОСЦИЛЛЯТОР С ТРЕНИЕМ И КЛАССИЧЕСКОЕ ПРЕДСТАВЛЕНИЕ 475

\section{4. МАРГИНАЛЬНОЕ РАСПРЕДЕЛЕНИЕ ВОЗБУЖДЕННОГО СОСТОЯНИЯ И ВЕРОЯТНОСТЬ ПЕРЕХОДА ОСЦИЛЛЯТОРА С ТРЕНИЕМ}

Рассмотрим интегралы движения $\hat{A}(t), \hat{A}^{\dagger}(t)$, которые могут быть найдены из системы уравнений для преобразования Боголюбова

$$
\begin{aligned}
\hat{A}(t) & =u \hat{a}(t)+v \hat{a}^{\dagger}(t), \\
\hat{A}^{\dagger}(t) & =v^{*} \hat{a}(t)+u^{*} \hat{a}^{\dagger}(t),
\end{aligned}
$$

где коэффициенты $u, v$, которые не зависят от времени, удовлетворяют равенству

$$
|u|^{2}-|v|^{2}=1,
$$

а $a(t), a^{\dagger}(t)$ - линейные интегралы движения для квантового осциллятора с трением $(32),(33)$. Интегралы движения $\hat{A}(t), \hat{A}^{\dagger}(t)$ определены условием, что оператор $\hat{A}^{\dagger}(t=0)$ порождает из основного состояния осциллятора без трения его возбужденные состояния. Решив систему уравнений для случая $t=0$, получим, что коэффициенты $u, v$ равны соответственно ( $\Omega$ определяется уравнением $(11))$

$$
\begin{aligned}
& u=\frac{1+\Omega-i \gamma}{2 \sqrt{\Omega}}, \\
& v=\frac{1-\Omega-i \gamma}{2 \sqrt{\Omega}} .
\end{aligned}
$$

Тогда оператор $A(t)$ равен

$$
\hat{A}(t)=\frac{i}{\sqrt{2}}(\tilde{\varepsilon} p-\dot{\tilde{\varepsilon}} \exp (2 \gamma t) q)
$$

где

$$
\tilde{\varepsilon}=u \varepsilon-v \varepsilon^{*},
$$

а функция $\varepsilon(t)$ имеет вид (10). Что касается вновь введенной функции $\tilde{\varepsilon}(t)$, которая зависит от времени, то она удовлетворяет начальным условиям

$$
\tilde{\varepsilon}(0)=1, \quad \dot{\tilde{\varepsilon}}(0)=i
$$

и имеет вронскиан (9). Повторяя выгисления, сделанные в работе [21], мы получаем волновые функции для возбужденного состояния осциллятора с трением

$$
\widetilde{\Psi}_{n}(q, t)=\frac{1}{\sqrt[4]{\pi} \sqrt{\tilde{\varepsilon}}}\left(\frac{\tilde{\varepsilon}^{*}}{2 \tilde{\varepsilon}}\right)^{\frac{n}{2}} \frac{1}{\sqrt{n !}} \exp \left(\frac{i \dot{\tilde{\varepsilon}} e^{2 \gamma t}}{2 \tilde{\varepsilon}} q^{2}\right) H_{n}\left(\frac{q}{\sqrt{\tilde{\varepsilon} \tilde{\varepsilon}^{*}}}\right) .
$$

Зная волновые функции и повторяя вычисления, сделанные во втором разделе, мы находим маргинальное распределение для возбужденного состояния

$$
\begin{aligned}
& \widetilde{w}_{n}(X, \mu, \nu, t)=\widetilde{w}_{0}(X, \mu, \nu, t) \frac{1}{2^{n} n !} H_{n}^{2}\left(\frac{X}{\sqrt{\tilde{\varepsilon} \tilde{\varepsilon}^{*}\left(\tilde{a}^{2}+\tilde{b}^{2}\right)}}\right), \\
& \widetilde{w}_{0}(X, \mu, \nu, t)=\frac{1}{\sqrt{\pi \tilde{\varepsilon} \tilde{\varepsilon}^{*}\left(\tilde{a}^{2}+\tilde{b}^{2}\right)}} \exp \left(-\frac{X^{2}}{\tilde{\varepsilon} \tilde{\varepsilon}^{*}\left(\tilde{a}^{2}+\tilde{b}^{2}\right)}\right),
\end{aligned}
$$


где

$$
\begin{gathered}
\tilde{a}=\frac{\exp (2 \gamma t) \nu\left(\tilde{\varepsilon}^{*} \dot{\tilde{\varepsilon}}+\tilde{\varepsilon} \dot{\tilde{\varepsilon}}^{*}\right)}{2 \tilde{\varepsilon} \tilde{\varepsilon}^{*}}+\mu, \\
\tilde{b}=\frac{\nu}{\tilde{\varepsilon} \tilde{\varepsilon}^{*}} .
\end{gathered}
$$

Легко увидеть, что при $t=0$ данное маргинальное распределение совпадает с уже упоминавшимся маргинальным распределением гармонического осциллятора. Вероятность перехода из $n$-го состояния в $m$-е возбужденное состояние простого гармонического осциллятора может быть получена как из известной формулы для вероятности путем интегрирования начальной и конечной волновых функций, так и с помощью формулы для вероятности путем интегрирования начальных и конечных маргинальных распределений (формула была дана в работе [25]). Данная вероятность перехода может быть представлена как

$$
P(n \rightarrow m)=\frac{n !}{m !} \frac{2}{\sqrt{2+\varepsilon \varepsilon^{*}+\dot{\varepsilon} \dot{\varepsilon}^{*} e^{4 \gamma t}}}\left[P_{\frac{m+n}{2}}^{\frac{m-n}{2}}\left(\frac{2}{\sqrt{2+\varepsilon \varepsilon^{*}+\dot{\varepsilon} \dot{\varepsilon}^{*} e^{4 \gamma t}}}\right)\right]^{2}, \quad m>n
$$

где $P_{k}^{l}(x)$ - присоединенные полиномы Лежандра. Найденная вероятность перехода хорошо согласуется с результатом работы [22] для частного случая $f(t)=e^{-\frac{t}{\tau}}, g(t)=e^{\frac{t}{\tau}}$ (в нашем случае $\tau=\frac{1}{2 \gamma}$ ). Аналогичный интеграл для осциллятора с переменной частотой был вычислен в [28]. Вероятность остаться в нулевом состоянии имеет вид

$$
\begin{aligned}
& P(0 \rightarrow 0)=\frac{2}{\sqrt{2+\tilde{\varepsilon} \tilde{\varepsilon}^{*}+\dot{\tilde{\varepsilon}} \dot{\tilde{\varepsilon}}^{*} e^{4 \gamma t}}}= \\
& =\frac{2 \Omega}{\sqrt{2 \Omega^{2}+e^{2 \gamma t}\left(1-\gamma^{2} \cos 2 \Omega t-\gamma \Omega \sin 2 \Omega t\right)+e^{-2 \gamma t}\left(1-\gamma^{2} \cos 2 \Omega t+\gamma \Omega \sin 2 \Omega t\right)}} .
\end{aligned}
$$

K сожалению, эта вероятность перехода была неправильно вычислена в статье [23] (см. формулу (17a)), но два частных случая, которые были разобраны в этой статье, когда $\gamma \ll 1, \Omega \approx 1, \Omega t=0$ и $\gamma \ll 1, \Omega t=\pi[23]$ (формулы (18a) и $(18 \mathrm{~b})$ ), оказались правильными.

\section{5. ВЫЧИСЛЕНИЕ МАРГИНАЛЬНОГО РАСПРЕДЕЛЕНИЯ С ПОМОЩЬЮ ПОДСТАНОВКИ}

Кратко опишем еше один способ получения маргинальных распределений осциллятора с трением. Маргинальные распределения (20) и (53) могут быть найдены с помощью подстановки параметров $\mu \rightarrow \mu(t), \nu \rightarrow \nu(t)$, которые зависят от времени. Данный метод был предложен в $[10,25]$. В этих работах было показано, что для нестационарного осциллятора, если известно начальное маргинальное распределение при $t=0$

$$
w(X, \mu, \nu, t=0)=w_{0}(X, \mu, \nu)
$$

маргинальное распределение во времена $t \neq 0$ будет иметь форму

$$
w(X, \mu, \nu, t)=w_{0}(X, \mu(t), \nu(t))
$$


где была сделана замена

$$
\nu(t)=\frac{\dot{\varepsilon}-\dot{\varepsilon}^{*}}{2 i} \nu+\frac{\varepsilon-\varepsilon^{*}}{2 i} \mu
$$

$$
\mu(t)=\frac{\dot{\varepsilon}+\dot{\varepsilon}^{*}}{2} \nu+\frac{\varepsilon+\varepsilon^{*}}{2} \mu .
$$

В случае осциллятора с трением функции $\varepsilon(t), \varepsilon^{*}(t)$ понимаются как $\varepsilon\left(t\left(t^{\prime}\right)\right), \varepsilon^{*}\left(t\left(t^{\prime}\right)\right)$, а точка обозначает производную по переменной $t^{\prime}$. Легко видеть, что формулы (17) и (20) могут быть получены с помощью указанной подстановки.

\section{6. ЗАКЛЮЧЕНИЕ}

Результатом проведенного анализа является введение положительной, нормируемой функции распределения (маргинального распределения), удовлетворяюшей уравнению эволюции типа Фоккера-Планка и полностью задаюшей квантовое состояние частицы (матрицу плотности), которая позволяет описывать движение квантовой частицы с трением. В этом смысле описание квантового движения с трением сделано похожим на описание классического движения с трением, при котором взаимодействие частицы с термостатом описывается положительной функцией распределения. В рамках предложенного классического представления найдены функции распределения, описываюшие различные полные наборы квантовых состояний, а именно, когерентные состояния и дискретные фоковские состояния осциллятора с трением. Исходя из вышеприведенных вычислений можно сделать вывод, что маргинальное распределение, которое используется для классического описания квантового трения, можно получить с помощью нескольких различных способов. Чтобы вычислить маргинальное распределение, мы использовали метод производящей функции. Применяя этот метод, мы избежали вычисления функции Вигнера для системы. В данной статье мы показали, как получить такое же выражение маргинального распределения из альтернативного уравнения Шредингера. Также была предложена подстановка для получения аналогичного выражения маргинального распределения из известного начального (при $t=0)$ маргинального распределения. Были рассмотрены интегралы движения для маргинального распределения.

Как мы показали, вероятность перехода между дискретными уровнями гармонического осциллятора, возбужденного квантовым трением, может быть получена в рамках классического представления квантового состояния. Данная вероятность перехода может быть вычислена с помощью интеграла перекрытия начального и конечного маргинальных распределений [25]. Вклад в вероятность перехода учитывает влияние классической траектории из начального состояния в данной системе отсчета в конечное состояние, рассмотренное с точки зрения ансамбля систем отсчета в фазовом пространстве, которые характеризуются параметрами $\mu$ и $\nu$. Вероятность перехода для двух маргинальных распределений, которые описывают дискретные состояния осциллятора с трением и выражаются через функцию Гаусса и полиномы Эрмита, может быть получена через присоединенные полиномы Лежандра.

Работа поддержана Российским фондом фундаментальных исследований. 


\section{Список литературы}

[1] R. J. Glauber. Coherence and quantum detection. In: Rend. Scoula Intern. Fis. E. Fermi XLII. 1967. N. Y.: Acad. Press., 1969. P. 32.

[2] P. Ullersma. Physica. 1966. V. 32. № 1. P. 27.

[3] I. R. Senitzky. Phys. Rev. 1960. V. 119. № 2. P. 670.

[4] M.D. Kostin. J. Chem. Phys. 1983. V. 57. № 9. P. 3589.

[5] H.D. Doebler, G. A. Goldin. Phys. Lett. A. 1992. V. 162. P. 397.

[6] V. V. Dodonov, S. S. Mizrahi. Ann. Phys. 1995. V. 237. P. 226.

[7] P. Caldirola. Nuovo Cimento. 1941. V. 18. № 9. P. 393.

[8] E. Kanai. Progr. Theor. Phys. 1948. V. 3. № 4. P. 440.

[9] S. Mancini, V. I. Man’ko, P. Tombesi. Phys. Lett. A. 1996. V. 213. № 1; Found. Phys. (in print).

[10] O. V. Man'ko. J. Russ. Laser Res. New York: Plenum Press, 1996. V. 17. P. 439; Phys. Lett. A. (in print).

[11] V. I. Man'ko. Energy levels of quantum system in classical formulation of quantum mechanics. In: Proceedings of APCTP Conference (Seoul, June 1996). World Scientific, 1997 (in print); J. Russ. Laser Res. 1996. V. 17. P. 579.

[12] K. Vogel, H. Risken. Phys. Rev. A. 1989. V. 40. P. 2847.

[13] K. E. Cahill, R. J. Glauber. Phys. Rev. 1969. V. 177. P. 1882.

[14] E. Wigner. Phys. Rev. 1932. V. 40. P. 749.

[15] R. J. Glauber. Phys. Rev. Lett. 1963. V. 10. P. 84.

[16] E. C. G. Sudarshan. Phys. Rev. Lett. 1963. V. 10. P. 277.

[17] K. Husimi. Proc. Phys. Math. Soc. Japan. 1940. V. 23. P. 264

[18] Y. Kano. J. Math. Phys. 1986. V. 6. P. 1913.

[19] V. G. Bagrov, V. V. Belov, A. Y. Trifonov. Ann. Phys. 1996. V. 246. P. 231.

[20] В. В. Белов, В. П. Маслов. Докл. АН СССР. 1989. Т. 305. С. 574.

[21] V. V. Dodonov, V. I. Man'ko. Phys. Rev. A. 1979. V. 20. № 2. P. 550.

[22] L. F. Landovitz, A. M. Lezine, W. M. Schreiber. J. Math. Phys. 1980. V. 21. № 8. P. 2159.

[23] P. Croxson. Phys. Rev. A. 1994. V. 49. № 1. P. 588.

[24] В. В. Додонов, В.И. Манько. Тр. ФИАН. Т. 183. М.: Наука, 1987; V. V. Dodonov, V. I. Man'ko. Invariants and evolution of nonstationary quantum system. In: Proceedings of the Lebedev Physical Institute. 183. Ed. M.A. Markov. N. Y.: Nova Science, 1989.

[25] V. I. Man'ko. Quantum mechanics and classical probability theory. In: Proceedings of International conference "Symmetries in Science VIII", Bregenz (Austria). August 1996. Ed. B. Gruber (to be published by Plenum Press, 1997).

[26] П. Ермаков. Университетские известия (Киев). 1880. Т. 20. № 9. С. 1.

[27] H. R. Lewis, W. B. Riesenfeld. J. Math. Phys. 1969. V. 10. P. 1458.

[28] I. A. Malkin, V. I. Man'ko, D. A. Trifonov. Phys. Rev. D. 1970. V. 2. P. 1371.

\section{V.I. Man'ko, S.S. Saphonov \\ QUANTUM DAMPED OSCILLATOR AND CLASSICAL FORMULATION OF QUANTUM MECHANICS}

Поступила в редакцию 26.II.1997 г., после доработки 25.IV.1997 г.

The quadrature marginal distribution for quantum damped oscillator described by the Caldirola-Kanai model with the constant frequency is introduced in a frame of a classical-like formulation of quantum mechanics based on a symplectic tomography scheme. The marginal distribution for coherent states and Fock states of the damped oscillator expressed explicitly in terms of Gaussian and Hermite polynomials. 